\title{
Brain functional connectivity in unconstrained walking with and without an exoskeleton
}

\author{
Junhua Li (李俊华), Senior Member, IEEE, Nitish Thakor, Life Fellow, IEEE, \\ and Anastasios Bezerianos, Senior Member, IEEE
}

\begin{abstract}
An exoskeleton is utilized to effectively restore the motor function of amputees' limbs and is frequently employed in motor rehabilitation training during convalescence. Understanding of exoskeleton impact on the brain is required in order to better and more efficiently use the exoskeleton. Almost all previous studies investigated the exoskeleton effect on the brain in a situation with constraints such as predefined walking speed, which could lead to findings differed from that obtained in an unconstrained situation. We, therefore, performed an experiment of unconstrained walking with and without an exoskeleton. Both individual connections and graph metrics were explored and compared among walking conditions. We found that low-order functional connections and associated high-order functional connections mainly between the left centroparietal region and right frontal region were significantly different among walking conditions. Generally speaking, connective strength was enhanced in LOFC and was decreased in aHOFC when assistant force was provided by the exoskeleton. Further, we proposed connection length investigation and revealed the large majority of these connections were long-distance connectivity. Graph metric investigation discovered higher connectivity clustering in the walking with low exoskeleton-aided force compared to the walking without the exoskeleton. This study expanded the existing knowledge of the effect of exoskeleton on the brain and is of implications on new exoskeleton development and exoskeleton-aided rehabilitation training.
\end{abstract}

Index Terms-Low-Order Functional Connectivity, (Associated) High-Order Functional Connectivity, Graph Metric, Unconstrained Walking, EEG, Exoskeleton.

\section{INTRODUCTION}

A MBULATION is one of the most important functions for the human in daily life. Impairment in ambulation would significantly affect the living and work, as well as causing society burden. Varying incidents could

This work was supported by the National Natural Science Foundation of China under Grant 61806149.

Junhua $\mathrm{Li}$ is with the School of Computer Science and Electronic Engineering, University of Essex, Colchester, CO4 3SQ, UK, also with the Laboratory for Brain-Bionic Intelligence and Computational Neuroscience, Wuyi University, Jiangmen, 529020, China, and also with the Centre for Multidisciplinary Convergence Computing, School of Computer Science and Engineering, Northwestern Polytechnical University, Xi'an, 710072, China.

Nitish Thakor and Anastasios Bezerianos are with the N.1 Institute for Health, National University of Singapore, Singapore, 117456, Singapore.

Corresponding author: Junhua Li (e-mail: juhalee.bcmi@gmail.com, junhua.li@essex.ac.uk) result in disabled lower limbs or complete loss of limbs. For example, 168 out of 284 motorcycle accident victims suffered from lower limb injuries according to the report in [1]. To release the impact of lower limb impairment, an exoskeleton is usually utilized to help restore movement function by two different manners. For those who temporarily lose ambulant function and could recover through rehabilitation training, an exoskeleton is considered as a good assistant device for improving the effectiveness of rehabilitation training and expediting the recovery of motor function [2], [3], [4], [5]. If the motor function of the lower limb cannot be recovered by the rehabilitation training, the other manner replacing the impaired limb is employed. An exoskeleton is used to restore motor function by either augmenting the ambulant capability of the impaired limb or replacing the amputated limb in the case of a complete loss of the entire limb [6], [7], [8]. For either manner, the attached exoskeleton would impose an impact on the human brain, which should be investigated in order to better and more effectively use the exoskeleton.

Up to now, a lot of efforts have been paid to understand the effect of an exoskeleton on the brain. Similar to the neural mechanism of contra-lateral control from the brain to upper limbs, lower limbs are controlled by contralateral brain hemispheres although the regions controlling respective lower limbs are closer to the midline of the brain [9]. According to the previous investigations, we have known that power spectral densities (PSD) extracted from the EEG recordings under different walking conditions were different [10], [11], [12], hemispherical asymmetry in the PSD was differential between the walking condition with an exoskeleton and the walking condition without an exoskeleton [13], the PSD was suppressed during walking compared to the upright standing [14], and the correlative relationship between brain activity and muscular contraction of the lower limb [15] and so forth. These differences among walking conditions have been utilized as features, based on which different walking conditions were successfully classified [10], [16], [17]. The difference between walking and standing was also observed in brain connectivity [18]. Brain connectivity would be changed when an external stimulus was imposed on people [19] or training was conducted [20]. If an exoskeleton was used to assist walking, what happens in brain connectivity? An answer was given in the study addressing brain connectivity topology relevant to walking with and without an exoskeleton in 2016 [21]. This study elucidated that 
the efficiency of brain organization was enhanced when assistant force was provided by an exoskeleton to facilitate walking compared to the walking condition without the assistance of the exoskeleton [21]. The study gave the first attempt to look insight into the brain inter-regional connectivity but the sample size in the study was small. A further study with the larger sample is required to corroborate the findings.

In recent years, high-order functional connectivity (HOFC) was proposed to explore the correlation between topographical profiles. From this perspective of HOFC, studies based on functional magnetic resonance imaging (fMRI) found that patients with mild cognitive impairment exhibited abnormal HOFC in the brain with reference to the brain connectivity representation of healthy people [22], [23], [24], [25], [26]. Besides the modality of fMRI, the HOFC was also applied to EEG modality [27]. This study found that the HOFC was changed from alertness state to drowsiness state in a driving experiment and demonstrated that the HOFC was able to provide complementary information to the low-order functional connectivity (LOFC) for assessing driving drowsiness [27]. The HOFC should be also explored in the walking experiment so as to reveal the high-order brain connectivity under different walking conditions. Furthermore, graph metric could be applied to the HOFC to understand organization efficiency in the high-order brain connectivity.

Last but not least, almost all previous studies investigating the effect of an exoskeleton on the human brain were performed in a constrained situation. For instance, a treadmill was commonly employed in the walking experiments due to the easier setup of the experiment and the relatively precious control of walking speed. However, these constraints could alter the behavior of walking as participants might change their normal daily walking style in order to fit with the constraints, which might bias the findings derived from such experiments. To eliminate such potential bias, an unconstrained situation is required in the experiment for walking investigation.

As we can see from the above description, investigation in the walking is not enough and further studies are required to either compensate the lacks or deepen the insights. Therefore, we, in this study, designed a walking experiment in the unconstrained situation and attempted to explore different walking conditions with and without an exoskeleton from both individual connections and graph metrics for LOFC, HOFC, and associated HOFC (aHOFC). According to the published literature [16], [11], [28], [10], alpha band and beta band were dominantly involved in walking. Hence, our investigation was focused on these two individual bands as well as the incorporative frequency band of alpha and beta bands (i.e. $8 \sim 30 \mathrm{~Hz}$ ). The details of the experiment setup, data processing, and methodologies including LOFC, HOFC, and graph metrics were sequentially described in the following sections. These sections were followed by the section of results, where the findings derived from this study were presented. These results were discussed in the next section.

\section{EXPERIMENT}

In this experiment, there were four different walking conditions: (1) Zero Force (ZF), participants performed walking with an exoskeleton but no assistant torque was provided to facilitate the walking; (2) Free Walk (FW), participants did not wear an exoskeleton and performed the walking; (3) Low Assistant Force (LAF), participants walked with an exoskeleton and low assistant torque was provided to facilitate the walking; and (4) High Assistant Force (HAF), participants walked with an exoskeleton with high assistant torque. The experiment was conducted in a level corridor approximately 21 meters long and 2.1 meters wide. Equipment and monitoring displayers were accommodated in a triple-deck trolley, which was located beside but maintained at a proper distance to the participant (please refer to Fig. 1 to have the overview of the experimental environment). The exoskeleton used in this experiment was a compact and wearable robotic system that was optimized based on the biomechanics of human gait to provide suitable assistant force for overground walking [29]. In the LAF walking condition, the impedance controller of the exoskeleton was set to $0.2 \mathrm{Nm} / \mathrm{deg}$ to provide low assistant force. It was $0.4 \mathrm{Nm} / \mathrm{deg}$ to provide high assistant force when performing the HAF walking condition [30].

During the walking, 62-channel EEG, one bipolar channel EOG, and four bipolar channels EMG were recorded using an ANT ASA-Lab system (ANT; Enschede, Netherlands). An additional EEG reference electrode was attached to the right earlobe. All channels were sychronized to record data at a sampling rate of $1,000 \mathrm{~Hz}$ with impedance kept below $10 \mathrm{k} \Omega$. In order to normalize EMG data for mitigating differences in the forcegenerating capacities of various muscles and variations across participants [31], [32], a reference EMG record was collected while the participants voluntarily contracted their muscles to the maximal extent (i.e., maximal voluntary contraction, MVC) before the recording for four walking conditions.

A total of 30 participants were recruited to attend this experiment. All are males in order to fit with the range of height and weight specified by the exoskeleton and the consideration of generally inequivalent physical strengths between males and females. Three of them were excluded from the following data analysis because of incomplete data recording. Based on the self-report, participants did not have any history of major lower limb injury or known neurological or locomotor deficits when they attended the experiment. They had normal vision or correctedto-normal vision, aged at the mean of 24 years and the standard deviation of 2.32 years. Their average body mass index (BMI) was $22.92 \pm 2.76$ (mean \pm standard deviation). Participants were instructed to walk normally similar to their daily walking without any restriction. They walked from one end to the other end of the 21-meter long corridor, counting one time, and repeated each walking condition three times. The study was approved by the 


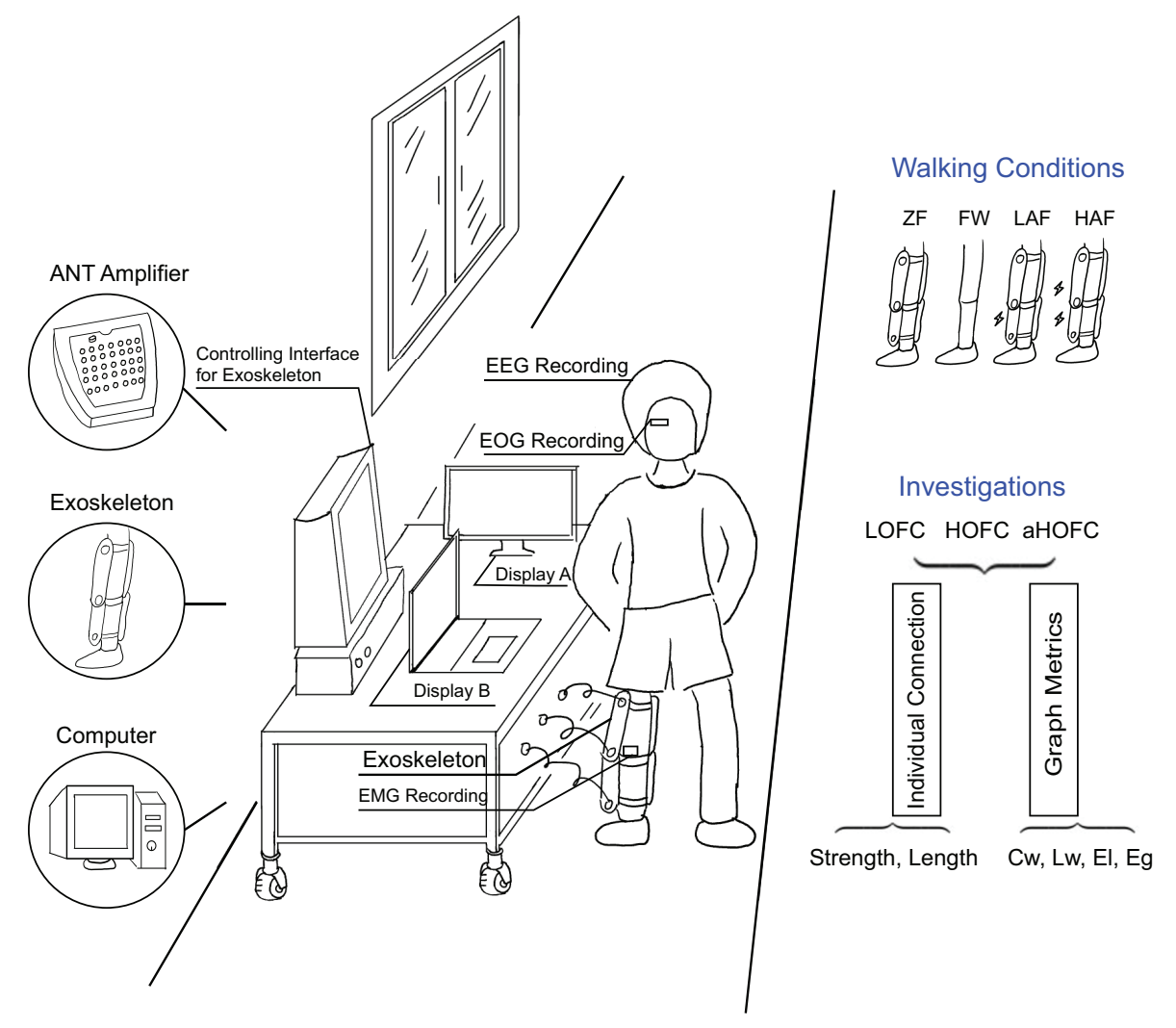

Fig. 1. Experimental environment. There are four walking conditions: (1) Zero Force (ZF), participants performed walking with an exoskeleton but no assistant torque was provided to facilitate the walking; (2) Free Walk (FW), participants did not wear an exoskeleton and performed the walking; (3) Low Assistant Force (LAF), participants walked with an exoskeleton and low assistant torque was provided to facilitate the walking; and (4) High Assistant Force (HAF), participants walked with an exoskeleton with high assistant torque. EEG, EOG, and EMG were simultaneously recorded from the participants.

Institutional Review Board of the National University of Singapore (NUS) and all participants provided written informed consent.

\section{Data Processing}

As the experiment was accomplished in an unconstrained situation, no gait cycle reference (e.g., heel strike) was recorded. We, thus, used EMG data to obtain the information of gait cycles. EMG data were first detrended and centered and then bandpass $(2 \sim 400 \mathrm{~Hz})$ filtered. After that, the filtered EMG was normalized using the reference record to be a form of a percentage of the MVC [31], [32]. A peak detection method was subsequently utilized to partition continuous recording into gait cycles based on the principle of that magnitude fluctuation pattern is similar across gait cycles although magnitude itself varies from one gait cycle to another [33]. A visual inspection was further conducted to adjust the gait cycle partition or to remove abnormal gait cycles for improving the precision of the partition. If the length of a gait cycle was one standard deviation shorter or longer than the average length, it was removed. The numbers of the remaining gait cycles were $65.1 \pm 16.7,63.3 \pm 11.3,62.0 \pm 13.5$, and 59.9 \pm 12.3 (mean \pm standard deviation) for $\mathrm{ZF}, \mathrm{FW}, \mathrm{LAF}$, and $\mathrm{HAF}$, respectively. The corresponding markers of gait cycles were stored for the following EEG partition.
Artifacts should be removed before EEG data analysis [34]. For EEG data, each channel was centered and then downsampled to $250 \mathrm{~Hz}$, which was followed by a bandpass $(0.5 \sim 45 \mathrm{~Hz})$ filter. An adaptive filtering method was used to mitigate the effect of eyes' movements on EEG [35] and a canonical correlation analysis-based method was used to reduce the EMG effect on the EEG [36]. Thereafter, independent component analysis (ICA) using the infomax algorithm was utilized to decompose the remaining EEG segments (continuous EEG was equally divided into two-second segments and abnormal segments were rejected using the procedure of segment rejection in the EEGLAB toolbox [37]) into signal sources (i.e., independent components). The independent components representing the remaining artifacts after preceding EOG and EMG artifact mitigation or the pulse interference were removed. The remaining independent components were then back-projected to reconstruct the continuous EEG signal. This reconstructed EEG signal was then partitioned into gait cycles according to the gait cycle markers that had been obtained using the EMG data. All EEG gait cycles were time-warped to be the same length. This was separately done for each participant. 
(A)

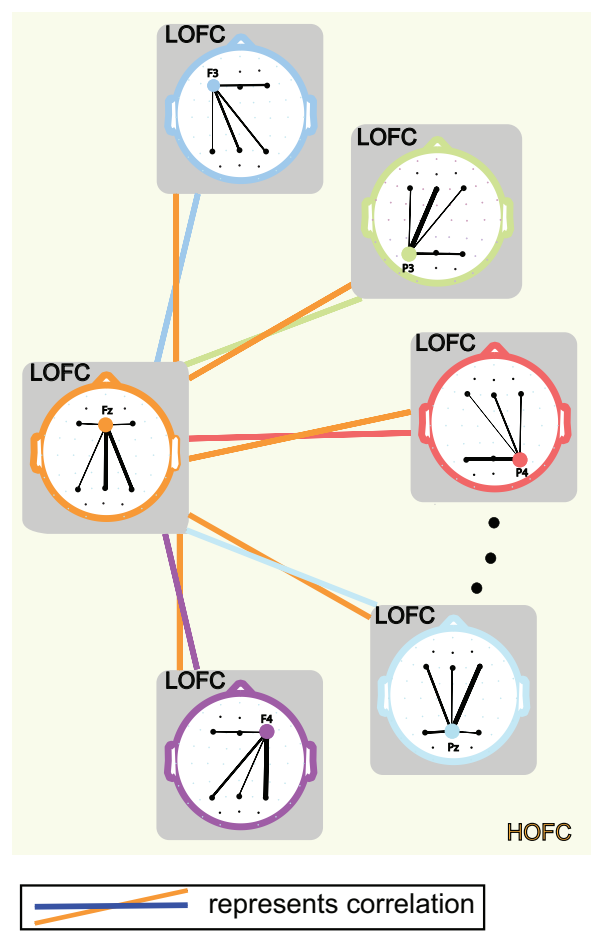

(B)

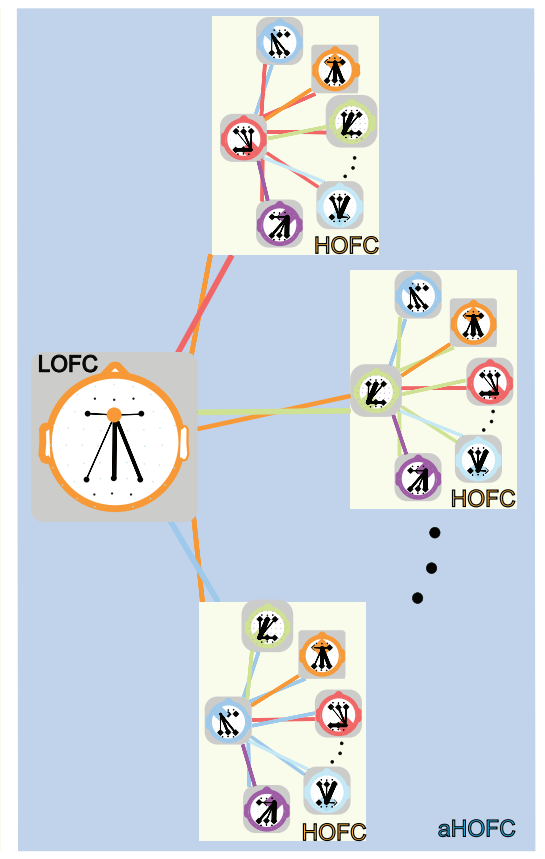

(C) Topographical

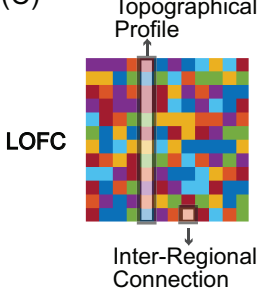

High-Order

Topographical Profile

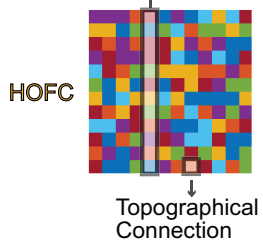

Associated

Topographical Profile

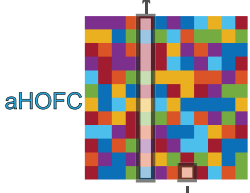

Associăted

Connection

Fig. 2. (A) High-order functional connectivity (HOFC) is obtained by correlation between topographical profiles. Low-order functional connectivity (LOFC) is inter-regional connectivity. (B) Associated high-order functional connectivity (aHOFC) is a measure assessing similarity between the topographical profile and the high-order topographical profile. (C) Illustration of connections and profiles of the LOFC, HOFC, and aHOFC.

\section{Methodologies}

\section{A. Low-Order Functional Connectivity}

In this study, partial directed coherence (PDC) was employed to estimate connective strengths for each pair of EEG channels. Specifically, relationships between EEG channels were modelled by multivariate autoregression as follows

$$
\left[\begin{array}{c}
x_{1}(t) \\
x_{2}(t) \\
\vdots \\
x_{K}(t)
\end{array}\right]=\sum_{r=1}^{q} A_{r}\left[\begin{array}{c}
x_{1}(t-r) \\
x_{2}(t-r) \\
\vdots \\
x_{K}(t-r)
\end{array}\right]+\left[\begin{array}{c}
w_{1}(t) \\
w_{2}(t) \\
\vdots \\
w_{K}(t)
\end{array}\right]
$$

where $X=\left[x_{1}, x_{2}, \cdots, x_{K}\right]^{\mathrm{T}}$ is EEG with $K$ channels, $A_{r}, r \in\{1,2, \cdots, q\}$ are $K \times K$ coefficient matrices for each lag $r, W=\left[w_{1}, w_{2}, \cdots, w_{K}\right]^{\mathrm{T}}$ is white uncorrelated noise. $q$ is order of the multivariate autoregression, which was determined by Akaike information criterion (AIC) [38],

$$
A I C(q)=2 \log (\operatorname{det}(\Sigma))+\frac{2 K^{2} q}{n_{\text {sample }}}
$$

where $\operatorname{det}(\Sigma)$ is the determinant of the covariance matrix of white noise $W(t)$, and $n_{\text {sample }}$ is the number of EEG samples. Once $A_{r}$ was estimated by the LevisonWiggins-Robinson algorithm [39], it can be transformed to frequency domain using Fourier transform, expressing as

$$
A(f)=\left.\sum_{r=1}^{q} A_{r} z^{-r}\right|_{z=e^{j 2 \pi f}}
$$

PDC from $j$ th channel to ith channel at frequency $f$ is defined as

$$
P D C_{i, j, f}=\frac{\left|a_{i j}(f)\right|}{\sqrt{\sum_{k=1}^{K} a^{*}{ }_{k j}(f) a_{k j}(f)}}
$$

where $a_{i j}$ is an element of $A(f)$, located at the intersection of the ith row and $j t h$ column. Asterisk stands for the transpose and complex conjugate operation. Connective strength for a certain band was obtained by averaging over PDCs of all frequencies within that band. In our case, three connectivity matrices $\mathbf{M}_{\alpha}, \mathbf{M}_{\beta}$, and $\mathbf{M}_{\alpha \beta}$ $\left(\mathbf{M}=\left[\mathbf{m}_{1}, \mathbf{m}_{2}, \cdots, \mathbf{m}_{K}\right] \in \mathbb{R}^{K \times K}, K\right.$ is 62 in our case) were obtained, which corresponded to alpha band $(8 \sim 12$ $\mathrm{Hz})$, beta band $(13 \sim 30 \mathrm{~Hz})$, and the incorporative band of these two bands $(8 \sim 30 \mathrm{~Hz})$, respectively. These matrices represent low-order functional connectivity.

\section{B. High-Order Functional Connectivity}

Each column $\mathbf{m}_{k}(k=1,2, \cdots, K)$ in the LOFC matrix $\mathbf{M}$ represents topographical profile for the $k$ th channel (see Fig. 2(C) for the illustration). High-order functional connectivity is calculated by Pearson's correlation between each pair of topographical profiles (see Fig. 2(A)). Let $\mathbf{m}_{i}$ and $\mathbf{m}_{j}$ denote topographical profiles for the $i$ th and $j$ th channels, respectively. Before calculating HOFC, elements represent self-connection (the element located in the diagonal of $\mathbf{M}$ ) and the connection to the counterpart 
channel are removed from the profile. After removal, the topographical profiles for the $i$ th and $j$ th channels are rewritten as

$\mathbf{m}_{i}^{-}=\left[m_{1, i}, \cdots, m_{i-1, i}, m_{i+1, i}, \cdots, m_{j-1, i}, m_{j+1, i}, \cdots, m_{K, i}\right.$

and

$\mathbf{m}_{j}^{-}=\left[m_{1, j}, \cdots, m_{i-1, j}, m_{i+1, j}, \cdots, m_{j-1, j}, m_{j+1, j}, \cdots\right.$

The dimensions of the vectors $\mathbf{m}_{i}^{-}$and $\mathbf{m}_{j}^{-}$are $K-2$. These vectors are respectively normalized by subtracting the mean and dividing by the standard deviation. The HOFC between these two topographical profiles is calculated by

$$
\begin{aligned}
\operatorname{HOFC}_{i, j} & =\frac{\left\langle\mathbf{m}_{i}^{-}, \mathbf{m}_{j}^{-}\right\rangle}{\left|\mathbf{m}_{i}^{-}\right|\left|\mathbf{m}_{j}^{-}\right|} \\
& =\frac{\Sigma_{k \in \Theta}\left(m_{k, i}-\bar{m}_{i}\right)\left(m_{k, j}-\bar{m}_{j}\right)}{\sqrt{\Sigma_{k \in \Theta}\left(m_{k, i}-\bar{m}_{i}\right)^{2}} \sqrt{\sum_{k \in \Theta}\left(m_{k, j}-\bar{m}_{j}\right)^{2}}}
\end{aligned}
$$

where $\Theta=\{1,2, \cdots, i-1, i+1, \cdots, j-1, j+1, \cdots, K\}$, $\bar{m}_{i}$ and $\bar{m}_{j}$ represent the means of $m_{k, i}$ and $m_{k, j}$ of the $i$ th and $j$ th topographical profiles. After the $H O F C_{i, j}$ of each pair of topographical profiles were calculated, they were assembled into the HOFC matrix. Each element in the HOFC matrix represents the similarity of the topographical profiles, called topographical connection. In this paper, $\mathbf{H}_{\alpha}, \mathbf{H}_{\beta}$, and $\mathbf{H}_{\alpha \beta}$ stand for the HOFC matrices corresponding to the alpha band, beta band, and the incorporative band, respectively.

\section{Associated High-Order Functional Connectivity}

Associated high-order functional connectivity (aHOFC) is a measure assessing similarity between the topographical profile and the high-order topographical profile (see Fig. 2(B)). It is calculated by Pearson's correlation between a column $\mathbf{m}_{i}^{-}=$ $\left[m_{1, i}, \cdots, m_{i-1, i}, m_{i+1, i}, \cdots, m_{j-1, i}, m_{j+1, i}, \cdots, m_{K, i}\right] \in$ $\mathbb{R}^{K-2}$ in $\mathbf{M}$ and a column $\mathbf{h}_{j}^{-}=$ $\left[h_{1, j}, \cdots, h_{i-1, j}, h_{i+1, j}, \cdots, h_{j-1, j}, h_{j+1, j}, \cdots, h_{K, j}\right] \quad \in$ $\mathbb{R}^{K-2}$ in $\mathbf{H}$. It is worth noting that the elements represent self-connection/self-profile-correlation and the connection/correlation to the counterpart channel/profile are removed before calculating aHOFC. Similar to the normalization performed in the topographical profiles, high-order topographical profiles are normalized before calculating aHOFC by

$$
{ }_{a H O F C} C_{i, j}=\frac{\left\langle\mathbf{m}_{i}^{-}, \mathbf{h}_{j}^{-}\right\rangle}{\left|\mathbf{m}_{i}^{-}\right|\left|\mathbf{h}_{j}^{-}\right|}
$$

\section{Connection Length}

We proposed to define the length of a connection between two channels based on physical locations of the channels and attempted to explore the lengths of the connections which were significantly different among walking conditions. The coordinates of all 62 channels are shown in the supplementary Table I. The connection length between the $i$ th channel and the $j$ th channel is defined as

$$
D_{i, j}=\sqrt{\left(X_{i}-X_{j}\right)^{2}+\left(Y_{i}-Y_{j}\right)^{2}+\left(Z_{i}-Z_{j}\right)^{2}}
$$

where $X, Y, Z$ are coordinates of the location of a channel. After the distances of all possible pairs of two channels were calculated according to Equation (9), the $K$ distance mean and median can be calculated. We used the distance mean and median to categorize connections. If a connection length was greater than the distance mean, the connection was considered as a mean-exceeding longdistance connection. Similarly, a connection was called median-exceeding long-distance connection if its length was greater than the distance median.

\section{E. Graph Metrics}

Besides the above connection-level measures, we employed graph metrics to characterize connectivity properties. In this study, we used weighted clustering coefficient $\left(C_{w}\right)$ and local efficiency $(E l)$ to investigate community properties of the connectivity network while we used weighted characteristic path length $\left(L_{w}\right)$ and global efficiency $(E g)$ to show the efficiency of information flow among brain regions [40]. To calculate these graph metrics, a sparsity threshold must be specified. We applied a wide range of sparsity form 0.1 to 0.4 with an incremental step of 0.01 instead of a certain sparsity [21], because there is no definitive manner to determine an optimal sparsity threshold, by which all spurious connections can be removed [40]. The final value of a graph metric was obtained by taking the integral of individual values of this metric over the above range of sparsity. The formulas of the graph metrics were found below.

The metric of weighted clustering coefficient reflects the prevalence of clustered connectivity around individual channels. Weighted clustering coefficient $C w_{i}$ for the $i$ th channel in a given connectivity network $\mathbf{M}$ with $K$ channels $\Lambda=\{1,2, \cdots, K\}$ is defined as

$$
\begin{aligned}
C w_{i}= & \left(\sum _ { \substack { j \neq i \\
h \neq i , h \neq j } } ( m _ { j , i } ^ { 1 / 3 } + m _ { i , j } ^ { 1 / 3 } ) \left(m_{h, i}^{1 / 3}+\right.\right. \\
& \left.\left.m_{i, h}^{1 / 3}\right)\left(m_{j, h}^{1 / 3}+m_{h, j}^{1 / 3}\right)\right) /\left(2 \left[\left(B^{\mathrm{T}}+B\right)_{i}\right.\right. \\
& \left.\left.\left(\left(B^{\mathrm{T}}+B\right)_{i}-1\right)-2 B_{i, i}^{2}\right]\right)
\end{aligned}
$$

where $B$ is the adjacency matrix, in which $B_{i j(i \neq j)}$ is set to 1 when connection exists between the $i$ th channel and $j$ th channel [41]. The wighted clustering coefficient $(C w)$ of entire connectivity network is the average over weighted clustering coefficients of all channels.

$$
C w=\frac{1}{K} \sum_{i \in \Lambda} C w_{i}
$$

Weighted characteristic path length from the $i$ th channel to the $j$ th channel is defined as the sum of the lengths of edges in the shortest path starting from the $i$ th channel and reaching the $j$ th channel. The length of edge is calculated as the reciprocal of connective strength 
$\left(d_{i, j}=1 / m_{i, j}\right)$. The weighted characteristic path length $L w$ is defined as the mean of shortest path lengths of each pair of channels in a connectivity network $\mathbf{M}$ with $K$ channels [42], [43].

$$
L w=\frac{1}{K(K-1)} \sum_{i \in \Lambda} \sum_{i \neq j \in \Lambda} \min \left\{d_{i, j}\right\}
$$

where $\min \left\{d_{i, j}\right\}$ is the shortest path length between the $i$ th channel and the $j$ th channel. This metric reflects functional integration. Global efficiency $E g$ is the average inverse shortest path length in a connectivity network $\mathbf{M}$ with $K$ channels [44].

$$
E g=\frac{1}{K(K-1)} \sum_{i \neq j \in \Lambda} \frac{1}{\min \left\{d_{i, j}\right\}}
$$

This metric reflects the global efficiency of parallel information transfer in the network, which is inversely related to the $L w$. Local efficiency $E l$ is the global efficiency only computed on the neighbourhoods of a given channel [44].

$$
E l=\frac{1}{K} \sum_{i \in \Lambda} E g(i)
$$

where $E g(i)$ is the global efficiency in the neighbourhoods of the $i$ th channel (the set of channels that directly connect to the $i$ the channel). This metric reflects the efficiency of the network on the local scale.
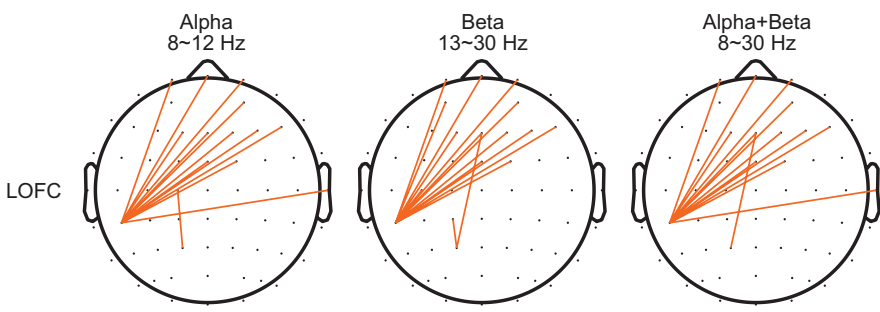

HOFC No Significant Connection
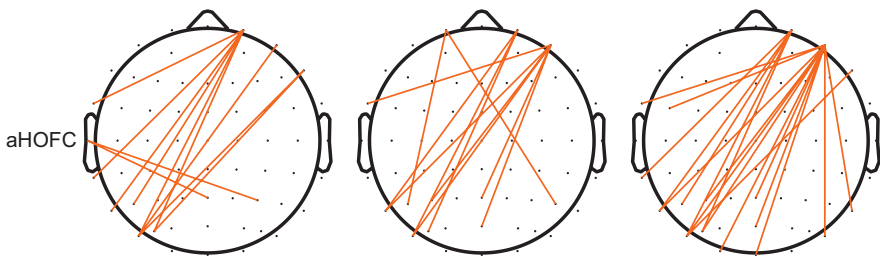

Fig. 3. Connections exhibiting significant differences among walking conditions for the low-order functional connectivity (LOFC), highorder functional connectivity (HOFC), and associated high-order functional connectivity (aHOFC).

\section{Results}

We investigated whether there was a significant difference among walking conditions in individual elements of connectivity matrices. One-way analysis of variance (ANOVA) with the factor of walking at four conditions revealed that there were 13,15 , and 13 connections exhibiting statistically significant differences among walking conditions in the $\mathrm{LOFC}_{\alpha}, \mathrm{LOFC}_{\beta}$, and $\mathrm{LOFC}_{\alpha \beta}$,
TABLE I

THE CONNECTIONS WITH SIGNIFICANT DIFFERENCES AMONG WALKING CONDITIONS IN THE $\mathrm{LOFC}_{\alpha}$

\begin{tabular}{ccccc}
\hline Connetction & Channel & Channel & F-value & p-value \\
\hline 1 & CP5 & Fp1 & 6.45 & 0.0005 \\
2 & CP5 & Fpz & 4.60 & 0.0046 \\
3 & CP5 & Fp2 & 6.52 & 0.0004 \\
4 & CP5 & Fz & 7.46 & 0.0001 \\
5 & CP5 & F4 & 5.35 & 0.0018 \\
6 & CP5 & FC2 & 5.43 & 0.0016 \\
7 & CP5 & T8 & 5.04 & 0.0027 \\
8 & CP5 & FF4 & 5.92 & 0.0009 \\
9 & CP5 & F1 & 5.21 & 0.0022 \\
10 & CP5 & F2 & 6.68 & 0.0004 \\
11 & CP5 & F6 & 5.06 & 0.0026 \\
12 & CP5 & FCz & 5.70 & 0.0012 \\
13 & P1 & C1 & 4.59 & 0.0047 \\
\hline
\end{tabular}

TABLE II

The CONNECTIONS WITH SIGNIFICANT DIFFERENCES AMONG WALKING CONDITIONS IN THE $\mathrm{LOFC}_{\beta}$

\begin{tabular}{ccccc}
\hline Connetction & Channel & Channel & F-value & p-value \\
\hline 1 & CP5 & Fp1 & 7.69 & 0.0001 \\
2 & CP5 & Fpz & 5.68 & 0.0012 \\
3 & CP5 & Fp2 & 6.66 & 0.0004 \\
4 & CP5 & Fz & 7.57 & 0.0001 \\
5 & P1 & Fz & 4.67 & 0.0042 \\
6 & CP5 & F4 & 5.90 & 0.0009 \\
7 & CP5 & FC2 & 6.22 & 0.0006 \\
8 & CP5 & C3 & 4.57 & 0.0048 \\
9 & P1 & CP1 & 4.77 & 0.0037 \\
10 & CP5 & AF3 & 4.61 & 0.0045 \\
11 & CP5 & AF4 & 7.17 & 0.0002 \\
12 & CP5 & F1 & 4.98 & 0.0029 \\
13 & CP5 & F2 & 6.90 & 0.0003 \\
14 & CP5 & F6 & 6.17 & 0.0007 \\
15 & CP5 & FCz & 5.47 & 0.0016 \\
\hline
\end{tabular}

respectively ( $p<0.005$, uncorrected). The distribution of these significantly different connections is depicted in the upper row of Fig. 3. The distribution patterns for each band are analogous, showing the connective relationship between the left centroparietal region and the medium, right frontal region. The specific channels involved in these connections with significant differences are listed along with statistical F-values and p-values in Table I, Table II, and Table III. There was no statistically significant

TABLE III

THE CONNECTIONS WITH SIGNIFICANT DIFFERENCES AMONG WALKING CONDITIONS IN THE $\mathrm{LOFC}_{\alpha \beta}$

\begin{tabular}{ccccc}
\hline Connetction & Channel & Channel & F-value & p-value \\
\hline 1 & CP5 & Fp1 & 7.44 & 0.0001 \\
2 & CP5 & Fpz & 5.49 & 0.0015 \\
3 & CP5 & Fp2 & 6.69 & 0.0004 \\
4 & CP5 & Fz & 7.63 & 0.0001 \\
5 & P1 & Fz & 4.67 & 0.0042 \\
6 & CP5 & F4 & 5.83 & 0.0010 \\
7 & CP5 & FC2 & 6.10 & 0.0007 \\
8 & CP5 & T8 & 4.54 & 0.0050 \\
9 & CP5 & FF4 & 6.96 & 0.0003 \\
10 & CP5 & F1 & 5.14 & 0.0023 \\
11 & CP5 & F2 & 6.96 & 0.0003 \\
12 & CP5 & F6 & 5.95 & 0.0009 \\
13 & CP5 & FCz & 5.56 & 0.0014 \\
\hline
\end{tabular}


topographical connection found in the HOFC. For the aHOFC, we observed 11, 11, and 18 associated connections with statistically significant differences among walking conditions in the $\mathrm{aHOFC}_{\alpha}, \mathrm{aHOFC}_{\beta}$, and $\mathrm{aHOFC}_{\alpha \beta}$, respectively (see the bottom row in Fig. 3). The distribution of these associated connections was also generally between the left centroparietal region and the right frontal region but more widespread in the centroparietal region with extension to the occipital region. The channels involved in these associated connections and their statistics are shown in Table IV, Table V, and Table VI. The posthoc paired t-test showed that connective strengths of significant connections were greater in the exoskeletonaided walking with low assistant force (LAF) compared to the other three walking conditions in the LOFC (see the t-test results in Table VII for the $\mathrm{LOFC}_{\alpha}$ and in supplementary Table II and Table III for the $\mathrm{LOFC}_{\beta}$ and $\mathrm{LOFC}_{\alpha \beta}$ ). The connective strengths of the most of associated connections in the alpha band were depressed when assistant force was provided by an exoskeleton compared to the condition without the assistant force, while the higher connective strength existed in the walking condition without an exoskeleton compared to the walking condition with an exoskeleton without assistant force at a few associated connections (see supplementary Table IV). For the beta band and incorporative band, the results were similar. Please find the details in supplementary Table V and Table VI.
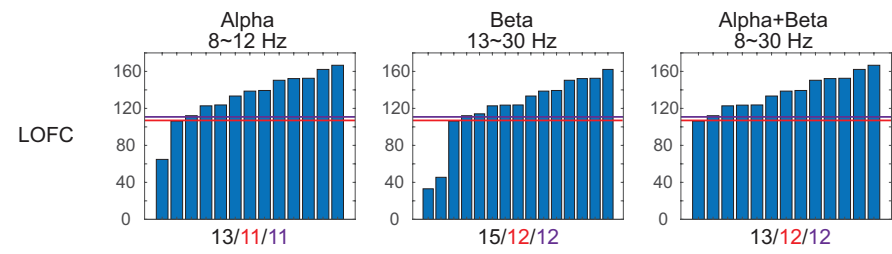

HOFC

No Significant Connection
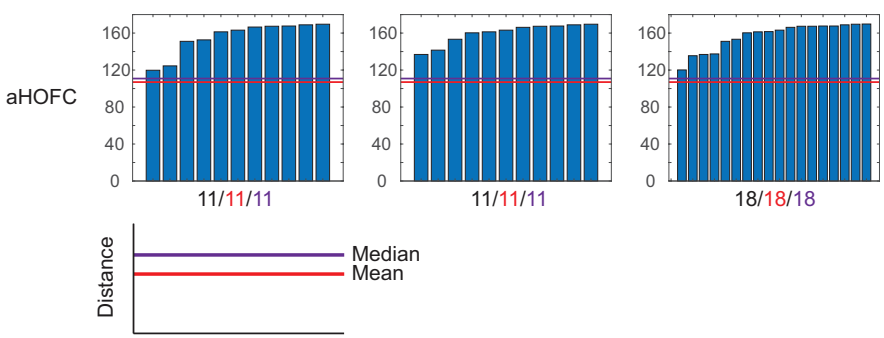

Fig. 4. Distances of the connections with significant differences among walking conditions. The horizontal red line represents the mean of distances of all pairs of two channels while the horizontal blue line represents the median of the distances. The numbers from left to right below each graph are the number of significant connections (exhibiting significant differences among walking conditions), the number of significant connections with length greater than the mean threshold (mean length is 106.98), and the number of significant connections with length greater than the median threshold (median length is 110.81), respectively.

The investigation of connection length showed that 11 out of 13 connections, 12 out of 15 connections, and 12 out of 13 connections were long-distance connections in the $\mathrm{LOFC}_{\alpha}, \mathrm{LOFC}_{\beta}$, and $\mathrm{LOFC}_{\alpha \beta}$, respectively (see Fig. 4).
TABLE IV

ThE CONNECTIONS WITH SIGNIFICANT DIFFERENCES AMONG WALKING CONDITIONS IN THE $\mathrm{AHOFC}_{\alpha}$

\begin{tabular}{ccccc}
\hline Connetction & Channel & Channel & F-value & p-value \\
\hline 1 & P7 & Fp2 & 5.56 & 0.0014 \\
2 & P5 & Fp2 & 5.13 & 0.0024 \\
3 & PO5 & Fp2 & 4.59 & 0.0047 \\
4 & FT7 & Fp2 & 4.56 & 0.0048 \\
5 & TP7 & Fp2 & 4.94 & 0.0030 \\
6 & PO7 & Fp2 & 5.71 & 0.0012 \\
7 & PO5 & F8 & 4.67 & 0.0042 \\
8 & PO7 & F8 & 4.92 & 0.0031 \\
9 & Pz & T7 & 4.69 & 0.0041 \\
10 & P4 & T7 & 4.65 & 0.0043 \\
11 & PO7 & AF8 & 4.71 & 0.0040 \\
\hline
\end{tabular}

TABLE V

THE CONNECTIONS WITH SIGNIFICANT DIFFERENCES AMONG WALKING CONDITIONS IN THE $\mathrm{AHOFC}_{\beta}$

\begin{tabular}{ccccc}
\hline Connetction & Channel & Channel & F-value & p-value \\
\hline 1 & P5 & Fp1 & 5.04 & 0.0027 \\
2 & P6 & Fp1 & 4.68 & 0.0041 \\
3 & P7 & Fp2 & 4.95 & 0.0030 \\
4 & PO5 & Fp2 & 4.69 & 0.0041 \\
5 & PO7 & Fp2 & 5.72 & 0.0012 \\
6 & P7 & AF8 & 4.81 & 0.0035 \\
7 & P3 & AF8 & 4.53 & 0.0050 \\
8 & Pz & AF8 & 4.71 & 0.0040 \\
9 & POz & AF8 & 5.00 & 0.0028 \\
10 & FT7 & AF8 & 4.64 & 0.0044 \\
11 & PO7 & AF8 & 5.23 & 0.0021 \\
\hline
\end{tabular}

This reflects that the connections differing among walking conditions were those who connected remote channels. All associated connections which exhibited significant differences in the ANOVA were long-distance connections for all explored bands (i.e., alpha band, beta band, and the incorporative band). Their lengths exceeded both the mean threshold (mean length is 106.98) and the median threshold (median length is 110.81) (see Fig. 4).

The investigation using graph metrics revealed that there was a significant difference in weighted clustering

TABLE VI

THE CONNECTIONS WITH SIGNIFICANT DIFFERENCES AMONG WALKING CONDITIONS IN THE $\mathrm{AHOFC}_{\alpha \beta}$

\begin{tabular}{ccccc}
\hline Connetction & Channel & Channel & F-value & p-value \\
\hline 1 & P7 & Fp2 & 5.44 & 0.0016 \\
2 & P5 & Fp2 & 4.83 & 0.0035 \\
3 & PO5 & Fp2 & 5.07 & 0.0026 \\
4 & TP7 & Fp2 & 4.77 & 0.0037 \\
5 & PO7 & Fp2 & 6.18 & 0.0007 \\
6 & PO7 & F8 & 4.56 & 0.0048 \\
7 & FC5 & AF8 & 4.82 & 0.0035 \\
8 & P7 & AF8 & 5.40 & 0.0017 \\
9 & P3 & AF8 & 4.81 & 0.0035 \\
10 & Pz & AF8 & 5.00 & 0.0028 \\
11 & P8 & AF8 & 4.84 & 0.0034 \\
12 & POz & AF8 & 5.55 & 0.0014 \\
13 & O1 & AF8 & 5.04 & 0.0027 \\
14 & Oz & AF8 & 4.56 & 0.0048 \\
15 & PO5 & AF8 & 4.91 & 0.0031 \\
16 & FT7 & AF8 & 4.91 & 0.0031 \\
17 & PO7 & AF8 & 5.92 & 0.0009 \\
18 & PO8 & AF8 & 4.76 & 0.0038 \\
\hline
\end{tabular}


TABLE VII

The POST-HoC T-Test RESUlts For The LOFC $\mathrm{C}_{\alpha}$

\begin{tabular}{|c|c|c|c|c|c|c|}
\hline Connection & $\begin{array}{c}\text { ZF versus FW } \\
\text { t-value ( } p \text {-value) }\end{array}$ & $\begin{array}{l}\mathrm{ZF} \text { versus LAF } \\
\text { t-value ( } \mathrm{p} \text {-value) }\end{array}$ & $\begin{array}{l}\mathrm{ZF} \text { versus HAF } \\
\text { t-value ( } p \text {-value) }\end{array}$ & $\begin{array}{l}\text { FW versus LAF } \\
\text { t-value (p-value) }\end{array}$ & $\begin{array}{l}\text { FW versus HAF } \\
\text { t-value ( } \mathrm{p} \text {-value) }\end{array}$ & $\begin{array}{l}\text { LAF versus HAF } \\
\text { t-value (p-value) }\end{array}$ \\
\hline 1 & - & $-3.67 \uparrow(0.0011)$ & - & $-3.21 \uparrow(0.0035)$ & - & $2.84 \downarrow(0.0087)$ \\
\hline 2 & - & $-3.59 \uparrow(0.0013)$ & $-2.24 \uparrow(0.0340)$ & $-2.51 \uparrow(0.0186)$ & - & - \\
\hline 3 & - & $-3.21 \uparrow(0.0035)$ & - & $-3.31 \uparrow(0.0027)$ & - & $2.79 \downarrow(0.0097)$ \\
\hline 4 & - & $-3.67 \uparrow(0.0011)$ & - & $-3.18 \uparrow(0.0038)$ & - & $2.97 \downarrow(0.0063)$ \\
\hline 5 & - & $-3.11 \uparrow(0.0045)$ & - & $-2.19 \uparrow(0.0374)$ & - & $2.89 \downarrow(0.0076)$ \\
\hline 6 & - & $-3.52 \uparrow(0.0016)$ & - & $-2.25 \uparrow(0.0334)$ & - & $2.75 \downarrow(0.0108)$ \\
\hline 7 & - & $-2.83 \uparrow(0.0088)$ & - & $-2.72 \uparrow(0.0114)$ & - & $2.47 \downarrow(0.0206)$ \\
\hline 8 & - & $-3.45 \uparrow(0.0019)$ & - & $-2.36 \uparrow(0.0260)$ & - & $2.85 \downarrow(0.0085)$ \\
\hline 9 & - & $-3.61 \uparrow(0.0013)$ & - & $-2.43 \uparrow(0.0222)$ & - & $2.33 \downarrow(0.0280)$ \\
\hline 10 & - & $-3.69 \uparrow(0.0010)$ & - & $-2.45 \uparrow(0.0211)$ & - & $2.95 \downarrow(0.0066)$ \\
\hline 11 & - & $-2.65 \uparrow(0.0135)$ & - & $-2.65 \uparrow(0.0134)$ & - & $2.21 \downarrow(0.0364)$ \\
\hline 12 & - & $-3.62 \uparrow(0.0013)$ & - & $-2.42 \uparrow(0.0228)$ & - & $2.66 \downarrow(0.0132)$ \\
\hline 13 & - & $-2.90 \uparrow(0.0074)$ & - & $-2.42 \uparrow(0.0226)$ & - & $3.88 \downarrow(0.0006)$ \\
\hline
\end{tabular}

$\uparrow$ indicates the greater connective strength in the latter condition while $\downarrow$ indicates the less connective strength in the latter condition.

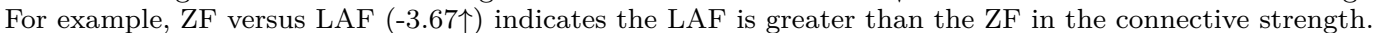

- indicates no significance at the significance level of 0.05 .

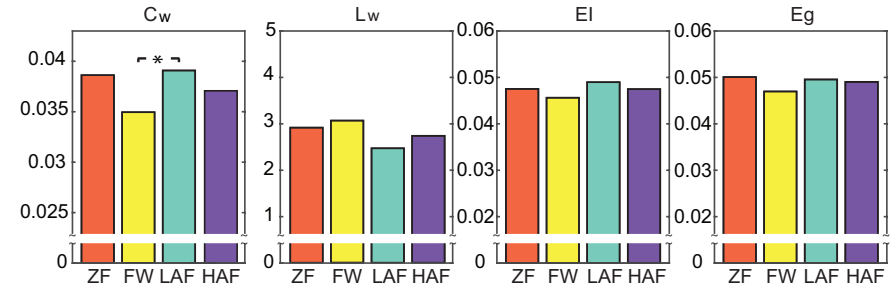

Fig. 5. Comparisons of graph metrics among walking conditions in the $\mathrm{LOFC}_{\beta}$. Asterisk indicates the significance level of the post-hoc t-test $(* p<0.05)$.

coefficients of the LOFC among walking conditions. Specifically, weighted clustering coefficient in the beta band for the LOFC was significantly different among walking conditions $(\mathrm{F}[3,104]=2.86, \mathrm{p}=0.0405$, uncorrected $)$. It was marginally significant in the alpha band for the LOFC $(\mathrm{F}[3,104]=2.59, \mathrm{p}=0.0567$, uncorrected $)$. For the incorporative band $(8 \sim 30 \mathrm{~Hz})$, there were significant differences among walking conditions $(\mathrm{F}[3,104]=2.77, \mathrm{p}=0.0453$, uncorrected). No other significant case was found in the metrics of Lw, El, and Eg for the LOFC. There was no significant case found in all metrics for the HOFC and aHOFC. The post-hoc t-test showed that the significant difference of $\mathrm{Cw}$ in the beta band for the $\mathrm{LOFC}$ was due to the significant difference between the walking without an exoskeleton (FW) and the walking with low assistant force provided by an exoskeleton (LAF) (see Fig. 5). In the case of the incorporative band, the significant difference was also derived from the significant difference between the FW and LAF (see supplementary Fig. 1).

\section{Discussions}

In this study, we designed an experiment in an unconstrained situation to explore brain functional connectivity at four kinds of walking. The unconstrained setting made participants not to be distracted by imposed constraints and not have to pay extra effort to fit with constraints. This could empower that the observations from such experiment are more congruent with what they should be and the findings could realistically describe the underlying neural mechanisms exerting in the walking. We explored both individual connections and graph metrics for the four walking conditions and revealed the differences among them. The exploration was not only performed in the LOFC but also performed in the HOFC and aHOFC. We found that the connections mainly linking between the left centroparietal region and the right frontal region were significantly different among walking conditions for the alpha band, beta band, and the incorporative band $(8 \sim 30 \mathrm{~Hz})$ in the LOFC and aHOFC, but not in the HOFC. Based on the investigation of connection length, we revealed that the large majority of significant connections in the LOFC were long-distance and all significant connections in the aHOFC were long-distance. The graph metric exploration showed that weighted clustering coefficients in the $\mathrm{LOFC}_{\beta}$ and $\mathrm{LOFC}_{\alpha \beta}$ were significantly different among walking conditions, which was attributed to the significant differences between the walking without an exoskeleton (FW) and the walking with low assistant force provided by an exoskeleton (LAF). There was no other significant case existing in other metrics for the LOFC and any metrics for the HOFC and aHOFC. The detailed discussions of the findings in this study are drawn below.

The connections with significant differences among walking conditions are very similar across the bands (i.e., alpha band, beta band, and the incorporative band) in the LOFC, leading to the analogous distribution pattern. In the aHOFC, the connections were also similar although the similarity between the distribution patterns across the bands was not as high as that appeared in the LOFC. These results demonstrated that the alpha and beta bands were equivalently involving in the walking in terms of inter-regional connectivity. The equivalent contribution to the walking was roughly in agreement with the observation that power spectral densities (PSD) of these two bands were concurrently changed relevant to walking [11], [28], [10] as well as the movements of upper limbs [45]. This might be due to that the alpha and beta bands are modulated to accomplish the largely overlapping part of 
the motor function. Our study showed that significantly different connections among walking conditions were those mainly linking between the left centroparietal region and the right frontal region, rather than the motor region. This might be because the connections in the motor region do not appear enough difference and the difference might be too small to be observed in the experiment. This possibility has been addressed in the paper investigating hemispherical asymmetry of the PSD relevant to the walking [13]. This paper reported the significantly asymmetric PSD appeared in the parietooccipital region, rather than motor region. We assumed that brain regions dominantly involving in the implementation of movements exerted similarly across walking conditions. The brain regions dominantly involving in the motor coordination and adjustment should exert more differently so as to meet the particular requirement of a specific type of walking. The similarity across the bands was also observed in the results of post-hoc t-test. The comparison pairs (e.g., ZF versus LAF) turning up statistical significance at one band were also statistically significant in the other two bands to a large extent (please refer to Table VII, supplementary Table II and Table III). For instance, the comparison pair ( $\mathrm{ZF}$ versus $\mathrm{LAF}$ ) showing statistical significance in the $\mathrm{LOFC}_{\alpha}$ was also significant in the $\mathrm{LOFC}_{\beta}$ and $\mathrm{LOFC}_{\alpha \beta}$. The direction ( $\mathrm{ZF}$ was less than LAF) was also the same across the bands. Such phenomenon was also approximately tenable in the aHOFC. These results derived from the post-hoc tests support the above assumption that alpha and beta bands were involved in the largely overlapping part of the motor function. Our study revealed that connective strengths were increased when low assistant force was provided to facilitate the walking compared to the walking without assistant force in the LOFC, but further enhanced assistant force (HAF) did not lead to more elevation in the connective strengths. Although the connective strengths at the walking condition with $\mathrm{HAF}$ were higher than that of the walking with the exoskeleton without assistant force, they were less than that of the walking with low assistant force (LAF). This demonstrated that higher assistant force might not always result in proper assistance for the walking. Appropriate assistant force which fits with the participant could be optimal to provide effective assistance for the walking. From the post-hoc t-test results of aHOFC, it can be seen that assistant force lowered the correlation between topographical profiles (a column in the LOFC matrix) and high-order topographical profiles (a column in the HOFC matrix) (see Fig. 2(C) for the illustration of profiles).

We proposed the investigation of connection length on the connections with the significant differences among walking conditions. The results of this investigation were intriguing. The large majority of connections with significant differences among walking conditions were longdistance in the LOFC and all the connections were longdistance in the aHOFC, which reflected that connective difference among walking conditions mainly existed in the long-distance connections in terms of individual connective strength. These long-distance connections might serve to convey information between remote brain regions, which is differential among walking conditions. When we took all connections and explored them as a whole using graph metrics, we found that the significant differences among walking conditions only appeared in the weighted clustering coefficient $(\mathrm{Cw})$ and this metric $\mathrm{Cw}$ in the walking condition with low assistant force (LAF) was greater than that in the walking condition without an exoskeleton (FW). However, our previous preliminary study found more significant cases such as in the case of characteristic path length [21]. This might be due to the small sample size (i.e., seven participants) in the previous preliminary study. The relatively large size of samples (i.e., 27 participants) in this study enhances the confidence of the findings. Although the less number of significant cases observed in this study, the results were compatible with the results showed in the previous preliminary study. In the $\mathrm{Cw}$ comparison between the walking condition with high assistant force (HAF) and the walking without an exoskeleton (FW), we did not find a statistically significant difference between them. This violated our initial hypothesis that high assistant force should push the change further towards the change direction from the FW to the LAF. The possible explanation might be the one we mentioned above. The higher assistant force might not always result in proper assistance for the walking. An appropriate assistant force which fits with the participant could be optimal to provide effective assistance for the walking. This point informs that an exoskeleton-aided study should consider physical characteristics (e.g., bodyweight and physical strength) of participants to set appropriate assistant force. This point also motivates a study, where more levels of assistant force are included to reveal the relationship between the level of the provided assistance and the brain reactions during walking.

As discussed above, the physical characteristics of participants could be critical to determine appropriate assistant force. We had considered the factors of physical strength, height, and weight in the criteria of participant recruitment but we did not particularly consider the relationship between these characteristics and the level of assistant force and quantified the assistant force to provide optimally appropriate force. This is a limitation of our study. In the statistical evaluation, multiple comparison correction was not applied to correct p-values because we would like to provide all original results of statistics as this was an exploratory study. We, for the first time, explored high-order functional connectivity for the walking with and without an exoskeleton in an unconstrained situation. Original p-values were provided in this paper so readers are able to have their own interpretation by using another threshold of the significance level. Another point we want to mention is volume conduction. To minimise the effect of volume conduction, we used partial directed coherence to estimate functional connectivity as it is insensitive to the volume conduction. Alternatively, transforming to source domain and then estimating functional connectivity can 
also dramatically eliminate the effect of volume conduction (a review of source connectivity analysis can be found in $[46])$.

\section{REFERENCES}

[1] J. Kortor, T. Kpela, and S. Popoola, "Lower limb disabilities following motorcyle crashes," Journal of Medicine in the Tropics, vol. 14, no. 2, pp. 147-150, 2012.

[2] J. C. Moreno, J. Figueiredo, and J. L. Pons, "Exoskeletons for lower-limb rehabilitation," in Rehabilitation Robotics. Elsevier, 2018, pp. 89-99.

[3] A. Esquenazi and M. Talaty, "Robotics for lower limb rehabilitation," Physical Medicine and Rehabilitation Clinics, vol. 30, no. 2, pp. 385-397, 2019.

[4] C. Krishnan, R. Ranganathan, Y. Y. Dhaher, and W. Z. Rymer, "A pilot study on the feasibility of robot-aided leg motor training to facilitate active participation," PloS one, vol. 8 , no. 10 p. e77370, 2013

[5] I. Díaz, J. J. Gil, and E. Sánchez, "Lower-limb robotic rehabilitation: literature review and challenges," Journal of Robotics, vol. 2011, 2011.

[6] R. J. Farris, H. A. Quintero, and M. Goldfarb, "Preliminary evaluation of a powered lower limb orthosis to aid walking in paraplegic individuals," IEEE Transactions on Neural Systems and Rehabilitation Engineering, vol. 19, no. 6, pp. 652-659, 2011.

[7] S. Mohammed, Y. Amirat, and H. Rifai, "Lower-limb movement assistance through wearable robots: state of the art and challenges," Advanced Robotics, vol. 26, no. 1-2, pp. 1-22, 2012.

[8] S. Wang, L. Wang, C. Meijneke, E. V. Asseldonk, T. Hoellinger, G. Cheron, Y. Ivanenko, V. L. Scaleia, and F. Sylos-labini, "Design and Control of the MINDWALKER Exoskeleton," IEEE transactions on neural systems and rehabilitation engineering, vol. 23, no. 2, pp. 277-86, 2015.

[9] F. Artoni, C. Fanciullacci, F. Bertolucci, A. Panarese, S. Makeig, S. Micera, and C. Chisari, "Unidirectional brain to muscle connectivity reveals motor cortex control of leg muscles during stereotyped walking," Neuroimage, vol. 159, pp. 403-416, 2017.

[10] M. Severens, M. Perusquia-Hernandez, B. Nienhuis, J. Farquhar, and J. Duysens, "Using actual and imagined walking related desynchronization features in a bci," IEEE transactions on neural systems and rehabilitation engineering, vol. 23 , no. 5 , pp. 877-886, 2014.

[11] J. Wagner, T. Solis-Escalante, P. Grieshofer, C. Neuper, G. Müller-Putz, and R. Scherer, "Level of participation in robotic-assisted treadmill walking modulates midline sensorimotor eeg rhythms in able-bodied subjects," Neuroimage, vol. 63 , no. 3, pp. 1203-1211, 2012.

[12] T. P. Luu, J. A. Brantley, S. Nakagome, F. Zhu, and J. L. Contreras-Vidal, "Electrocortical correlates of human levelground, slope, and stair walking," PloS one, vol. 12, no. 11, p. e0188500, 2017.

[13] J. Li, N. Thakor, and A. Bezerianos, "Unilateral exoskeleton imposes significantly different hemispherical effect in parietooccipital region, but not in other regions," Scientific reports, vol. 8, no. 1, p. 13470, 2018.

[14] M. Seeber, R. Scherer, J. Wagner, T. Solis-Escalante, and G. R. Muller-Putz, "EEG beta suppression and low gamma modulation are different elements of human upright walking," Frontiers in Human Neuroscience, vol. 8, no. July, pp. 1-9, 2014. [Online]. Available: http://www.frontiersin.org/ Human $\{\backslash$ \}Neuroscience/10.3389/fnhum.2014.00485/abstract

[15] Y. Hashimoto, J. Ushiba, A. Kimura, M. Liu, and Y. Tomita, "Correlation between eeg-emg coherence during isometric contraction and its imaginary execution," Acta Neurobiol Exp (Wars), vol. 70, no. 1, pp. 76-85, 2010.

[16] S. K. Goh, H. A. Abbass, K. C. Tan, A. Al-Mamun, N. Thakor, A. Bezerianos, and J. Li, "Spatio-spectral representation learning for electroencephalographic gait-pattern classification," IEEE Transactions on Neural Systems and Rehabilitation Engineering, vol. 26 , no. 9, pp. 1858-1867, 2018.

[17] J. Chai, G. Chen, P. Thangavel, G. N. Dimitrakopoulos, I. Kakkos, Y. Sun, Z. Dai, H. Yu, N. Thakor, A. Bezerianos et al., "Identification of gait-related brain activity using electroencephalographic signals," in 2017 8th International IEEE/EMBS
Conference on Neural Engineering (NER). IEEE, 2017, pp. $548-551$

[18] T. M. Lau, J. T. Gwin, and D. P. Ferris, "Walking reduces sensorimotor network connectivity compared to standing," Journal of neuroengineering and rehabilitation, vol. 11, no. 1, p. 14, 2014.

[19] G. Gálvez, M. Recuero, L. Canuet, and F. Del-Pozo, "Shortterm effects of binaural beats on eeg power, functional connectivity, cognition, gait and anxiety in parkinson's disease," International journal of neural systems, vol. 28, no. 05, p. 1750055, 2018

[20] V. Youssofzadeh, D. Zanotto, K. Wong-Lin, S. K. Agrawal, and G. Prasad, "Directed functional connectivity in frontocentroparietal circuit correlates with motor adaptation in gait training," IEEE Transactions on Neural Systems and Rehabilitation Engineering, vol. 24, no. 11, pp. 1265-1275, 2016.

[21] J. Li, G. Chen, P. Thangavel, H. Yu, N. Thakor, A. Bezerianos, and Y. Sun, "A robotic knee exoskeleton for walking assistance and connectivity topology exploration in eeg signal," in 2016 6th IEEE International Conference on Biomedical Robotics and Biomechatronics (BioRob). IEEE, 2016, pp. 1068-1073.

[22] Y. Zhang, H. Zhang, X. Chen, S.-W. Lee, and D. Shen, "Hybrid high-order functional connectivity networks using resting-state functional mri for mild cognitive impairment diagnosis," Scientific reports, vol. 7 , no. 1, p. 6530, 2017 .

[23] Y. Zhang, H. Zhang, X. Chen, M. Liu, X. Zhu, S.-W. Lee, and D. Shen, "Strength and similarity guided group-level brain functional network construction for mci diagnosis," Pattern Recognition, vol. 88, pp. 421-430, 2019.

[24] H. Zhang, X. Chen, F. Shi, G. Li, M. Kim, P. Giannakopoulos, S. Haller, and D. Shen, "Topographical information-based high-order functional connectivity and its application in abnormality detection for mild cognitive impairment," Journal of Alzheimer's Disease, vol. 54, no. 3, pp. 1095-1112, 2016.

[25] X. Chen, H. Zhang, S.-W. Lee, D. Shen, A. D. N. Initiative et al., "Hierarchical high-order functional connectivity networks and selective feature fusion for mci classification," Neuroinformatics, vol. 15, no. 3, pp. 271-284, 2017.

[26] X. Chen, H. Zhang, Y. Gao, C.-Y. Wee, G. Li, D. Shen, and A. D. N. Initiative, "High-order resting-state functional connectivity network for mci classification," Human brain mapping, vol. 37, no. 9, pp. 3282-3296, 2016.

[27] J. Harvy, N. Thakor, A. Bezerianos, and J. Li, "Betweenfrequency topographical and dynamic high-order functional connectivity for driving drowsiness assessment," IEEE Transactions on Neural Systems and Rehabilitation Engineering, vol. 27, no. 3, pp. 358-367, 2019.

[28] G. R. Müller-Putz, D. Zimmermann, B. Graimann, K. Nestinger, G. Korisek, and G. Pfurtscheller, "Eventrelated beta eeg-changes during passive and attempted foot movements in paraplegic patients," Brain research, vol. 1137, pp. 84-91, 2007.

[29] H. Yu, M. S. Cruz, G. Chen, S. Huang, C. Zhu, E. Chew, Y. S. $\mathrm{Ng}$, and N. V. Thakor, "Mechanical design of a portable kneeankle-foot robot," in Robotics and Automation (ICRA), 2013 IEEE International Conference on. IEEE, 2013, pp. 2183-2188.

[30] G. Chen, P. Qi, Z. Guo, and H. Yu, "Mechanical design and evaluation of a compact portable knee-ankle-foot robot for gait rehabilitation," Mechanism and Machine Theory, vol. 103, pp. $51-64,2016$

[31] G. Allison, R. Marshall, and K. Singer, "Emg signal amplitude normalization technique in stretch-shortening cycle movements," Journal of Electromyography and Kinesiology, vol. 3, no. 4, pp. 236-244, 1993.

[32] K. J. Netto and A. F. Burnett, "Reliability of normalisation methods for emg analysis of neck muscles," Work, vol. 26, no. 2, pp. 123-130, 2006.

[33] G. Kaur, A. S. Arora, and V. Jain, "Comparison of the techniques used for segmentation of emg signals," in Proceedings of the 11th WSEAS international conference on Mathematical and computational methods in science and engineering. World Scientific and Engineering Academy and Society (WSEAS), 2009 , pp. $124-129$.

[34] J. T. Gwin, K. Gramann, S. Makeig, and D. P. Ferris, "Removal of movement artifact from high-density eeg recorded during walking and running," Journal of neurophysiology, vol. 103, no. 6 , pp. $3526-3534,2010$ 
[35] P. He, G. Wilson, and C. Russell, "Removal of ocular artifacts from electro-encephalogram by adaptive filtering," Medical and biological engineering and computing, vol. 42, no. 3, pp. 407-412, 2004.

[36] J. Li, Y. Chen, F. Taya, J. Lim, K. Wong, Y. Sun, and A. Bezerianos, "A unified canonical correlation analysis-based framework for removing gradient artifact in concurrent eeg/fmri recording and motion artifact in walking recording from eeg signal," Medical \& Biological Engineering \& Computing, pp. 113, 2017.

[37] A. Delorme and S. Makeig, "Eeglab: an open source toolbox for analysis of single-trial eeg dynamics including independent component analysis," Journal of neuroscience methods, vol. 134, no. 1, pp. 9-21, 2004.

[38] H. Akaike, "A new look at the statistical model identification," Automatic Control, IEEE Transactions on, vol. 19, no. 6, pp. 716-723, 1974

[39] M. Morf, A. Vieira, D. T. Lee, and T. Kailath, "Recursive multichannel maximum entropy spectral estimation," IEEE Transactions on Geoscience Electronics, vol. 16, no. 2, pp. 8594, 1978.

[40] S. Achard and E. Bullmore, "Efficiency and cost of economical brain functional networks," PLoS Comput. Biol., vol. 3, no. 2, pp. e17-e17, 2007.

[41] S. Boccaletti, V. Latora, Y. Moreno, M. Chavez, and D.-U. Hwang, "Complex networks: Structure and dynamics," Physics reports, vol. 424, no. 4, pp. 175-308, 2006.

[42] D. J. Watts and S. H. Strogatz, "Collective dynamics of "smallworld'networks," Nature, vol. 393, no. 6684, pp. 440-442, 1998.

[43] M. Rubinov and O. Sporns, "Complex network measures of brain connectivity: uses and interpretations," NeuroImage, vol. 52, no. 3, pp. 1059-1069, 2010.

[44] V. Latora and M. Marchiori, "Efficient behavior of small-world networks," Phys. Rev. Lett., vol. 87, no. 19, p. 198701, 2001.

[45] G. Pfurtscheller, C. Neuper, D. Flotzinger, and M. Pregenzer, "EEG based discrimination between imagination of right and left hand movement," Electroencephaplography and clinical Neurophysiology, vol. 103, pp. 642-651, 1997.

[46] J.-M. Schoffelen and J. Gross, "Source connectivity analysis with meg and eeg," Human brain mapping, vol. 30, no. 6, pp. 1857-1865, 2009. 